\title{
Study design of a prospective, open-label study comparing increased infusion parameters of a $20 \%$ subcutaneous immunoglobulin (IgPro20) in patients with primary immunodeficiency
}

Mikhail Rojavin ( $\triangle$ mikhail.rojavin@cslbehring.com )

CSL Behring LLC https://orcid.org/0000-0002-9010-2865

Jutta Hofmann

CSL Behring AG

Michaela Praus

CSL Behring GmbH

\section{Methodology article}

Keywords: Immunoglobulin G replacement therapy, Hizentra ${ }^{\circledR}$, IgPro20, primary immunodeficiencies, infusion parameters, manual push, rapid push

Posted Date: November 6th, 2019

DOI: https://doi.org/10.21203/rs.2.16903/v1

License: (c) (i) This work is licensed under a Creative Commons Attribution 4.0 International License.

Read Full License 


\section{Abstract}

Background: Immunoglobulin G (IgG) replacement therapy can be administered subcutaneously (SCIG) using an infusion pump or by manual push. Both methods have shown similar serum IgG trough levels for the same total weekly/monthly dose as well as safety and tolerability profiles. The currently approved infusion parameters for the SCIG Hizentra ${ }^{\circledR}$ (IgPro20, CSL Behring, King of Prussia, PA, USA) in primary immunodeficiency (PID) in the United States are volumes of $\leq 25 \mathrm{~mL}$ and flow rates of $\leq 25 \mathrm{~mL} / \mathrm{h}$ per injection site. Previously, clinical studies and case reports had demonstrated use of higher infusion parameters than those approved, but safety and tolerability of these have not been systematically evaluated. In the absence of regulatory guidance, we have developed a novel prospective clinical study applying forced upward titration design to evaluate the safety and tolerability of high infusion parameters of IgPro20 in PID patients using pump-assisted and manual push techniques (NCT03033745).

Results: A total of 45 patients were planned for inclusion. Primary endpoints were defined as the proportion of patients successfully infusing certain infusion parameters (responder rate). The study included three cohorts ( $n=15$ planned per group): 1) Pump-assisted Volume Cohort with weekly infusions at volumes of 25, 40 and $50 \mathrm{~mL}$ per injection site; 2) Pump-assisted Flow Rate Cohort with weekly infusions at flow rates of 25, 50, 75 and $100 \mathrm{~mL} / \mathrm{h}$ per injection site, and 3) Manual Push Flow Rate Cohort with 2 to 7 infusions at flow rates 30,60 and $120 \mathrm{~mL} / \mathrm{h}$ per injection site. Each infusion parameter level was tested for 4 weeks, after which those who successfully infused at the current level (responders) were switched to the next level. Responder rate, safety and tolerability were assessed. Study results will be available in early 2020 .

Conclusions: This study applied a novel and rigorous prospective evaluation of individual safety and tolerability levels of pump-assisted and manual push SCIG at higher infusion parameters than currently approved in the United States and demonstrated their safety and tolerability.

\section{Background}

Primary immunodeficiency (PID) is primarily treated with immunoglobulin $\mathrm{G}(\mathrm{IgG})$ replacement therapy, administered either intravenously (IVIG) or subcutaneously (SCIG) $(1-4)$. In clinical practice, infusion pumps are used for SCIG administration, usually delivering up to $25 \mathrm{~mL}$ of IgG into the injection site, with weekly infusions taking up to 2 hours depending on individual dose and flow rate $(1,5)$. Although clinical experience has demonstrated that larger volumes and flow rates can be successfully administered using an infusion pump (3), safety and tolerability of higher infusion parameters have not been tested in a rigorous clinical study environment using forced upward titration design. An alternative to the infusion pump is the manual push technique, which offers self-administration by the patient in smaller, more frequent doses using a syringe and butterfly needle $(5,6)$. Manual push infusion is reported to be effective; have an acceptable safety profile; and provide treatment flexibility, independence, and satisfaction (7). Infusion times are much shorter (5 to 20 minutes) with manual push technique and fewer injection sites are used as compared with weekly pump-assisted infusions $(5,8,9)$. Both 
techniques, manual push and pump-assisted infusion, have previously shown similar serum IgG levels for the same dose and overall safety and tolerability profiles, although the comparative incidence of adverse events (AEs) varies between studies $(5,9-11)$. Therefore, the choice of SCIG administration using either pump-assisted or manual push infusion can be tailored according to the individual patient's profile and preferences $(6,12)$.

IgPro20 (Hizentra ${ }^{\circledR}$, CSL Behring, King of Prussia, PA, USA) is a ready-to-use formulation of polyvalent SCIG (highly purified [ $\geq 98 \%$ purity] $\lg G$ ) approved for the treatment of PID globally (13). Currently, a volume of up to $25 \mathrm{~mL}$ per injection site and a flow rate of up to $25 \mathrm{~mL} / \mathrm{h}$ per injection site are approved for IgPro20 in the United States for the treatment of PID (14). However, higher infusion volumes $(60 \mathrm{~mL}$ per injection site) and flow rates (>60 mL/h per injection site) of $20 \%$ SCIG products have been demonstrated to be well tolerated in both pediatric and adult patients, as well as in obese adults (9-11, 15). Higher infusion paremeters of SCIG products are important because, in general, patients on SCIG replacement therapy tend to prefer shorter infusion times (achievable with higher flow rates) and fewer needle sticks per infusion (requiring higher volume per individual injection site) $(16,17)$.

In order to fully characterize the current landscape regarding clinical studies conducted with pumpassisted and manual push infusion, a systematic search of the currently published literature was performed. The PubMed and EMBASE databases were searched from January 2009 until April 2019 for full text publications reporting actual treated patients with PID, using the following search terms: "subcutaneous immunoglobulin" or "subcutaneous immune globulin" or "SCIG" or "IGSC "manual administration" or "rapid push" or "manual" and "primary immunodeficiency" or "primary immune deficiency." In total, 15 studies were identified $(3,5,6,9-12,15,18-24)$, of which 8 studies met the inclusion criteria (Figure 1) $(5,6,9-12,15,20)$. Among the studies included in the analysis, a range of SCIG therapies were administered to patients with PID using manual push or pump-assisted techniques $(5,6,9-12,15,20)$. The duration of the treatment varied across the studies, ranging from 28 to 45.5 months. Mean IgG levels reported were similar between push and pump techniques and were also similar across the studies $(5,6,9-12,15,20)$. The comparative rate of infusion site reactions reported in the literature for both manual push and pump-assisted SCIG administration vary $(5,10,20)$. This is most likely due to differences in study design, scales, timepoints, and report methodology. Across the identified studies, the average flow rates using a manual push technique ranged from 12 to $60 \mathrm{~mL} / \mathrm{h}$ per injection site $(1,9,10,20)$ and for pump-assisted techniques at a volume of up to $50 \mathrm{~mL}$ per injection site (6) and an infusion flow rate of up to 20 to $50 \mathrm{~mL} / \mathrm{h}$ per injection site $(5,25-27)$. The studies reported no major safety concerns, as most AEs were local reactions mostly of mild nature. Although these clinical studies have demonstrated the safety and effectiveness of both the frequent use of higher infusion parameters than currently approved for IgPro20 in PID in the United States and the two infusion techniques (pumpassisted or manual push) in clinical practice, safety and tolerability of these has not been evaluated in a systematic manner in a prospective clinical trial where every patient is required to test their individual comfort limits regarding flow rate and/or volume per injection site. 
Of note, guidance from several major regulatory agencies is available for standard registration studies evaluating the efficacy, safety, and pharmacokinetics of IgG products in PID (28-30). However, such guidance and a standardized approach are lacking for studies evaluating infusion parameters of IgG products that are higher than those approved for existing IgG products. The present study was specifically designed to obtain safety and tolerability data of infusion parameters of IgPro20 higher than those currently approved in patients with PID for both pump-assisted and manual push techniques for regulatory purposes.

\section{Results}

As this study is ongoing, full results will be presented in the future.

\section{Discussion}

The present study aimed to address the gap in the existing landscape of standardized and/or recommended by regulatory guidance documents study designs that evaluate the safety and tolerability of IgG infusions in IgG replacement therapy. Namely, we have developed a trial of high infusion parameters testing weekly doses of IgPro20 of up to $100 \mathrm{~mL}$ per week, compared with an average United States dose of $50 \mathrm{~mL}$ (10 grams) per week. IgG replacement therapy (administered as either IVIG or SCIG) has become the cornerstone of treatment for PID, and as an immunomodulatory agent for an increasing number of autoimmune and inflammatory disorders (1-4). As such, there are well-characterized regulatory frameworks for evaluating efficacy, safety, or pharmacokinetics of these products, including specification that their evaluation in defined subpopulations such as pediatric, geriatric, and/or obese patients is an essential requirement (28-30). These recommendations facilitate the design of studies investigating these products. However, there are currently no such requirements or guidance on the investigation of various infusion parameters, such as volume and flow rate per injection site, or for various infusion techniques, such as pump-assisted or manual push administration. Lack of any regulatory guidance or data from other studies with forced upward titration design on this topic is a limitation to further improve the convenience of administration of SCIG therapies.

In pivotal (registration) IgG clinical studies, patients are allowed to test flow rate and volume parameters based on typical clinical practice within the range specified by study protocol and at levels generally considered tolerable to patients. However, these studies do not routinely investigate infusion parameters that are higher than those used conventionally, which may reduce the time needed for injection and the number of injection sites. The latter two characteristics reflect patients' preferences on SCIG and lead to a better health-related quality of life for patients with $\operatorname{PID}(16,17)$. A high, stable health-related quality of life is important both as a separate goal, especially in PID patients who need life-long IgG replacement therapy, and as a means of increasing patients' treatment compliance (31).

Another key question requiring discussion is how to define the minimum proportion of study patients achieving and tolerating a certain infusion parameter that would be acceptable for regulatory agencies to 
include the parameter as a generally recommended infusion regimen within the product labeling. This may vary by geographic region. For example, the European Union often defines the highest limits for injection volume or rate as that which is "individually tolerated," whereas United States prescribing information includes specific definitions for minimal and maximal injection volumes and flow rates, supported by clinical research. This is further complicated when the initial infusion parameter recommendations need to be updated based on the clinical evidences which shows possibility of alteration of the infusion parameters.

During interactions with the United states Food and Drug Administration regarding updates to the recommendations for IgPro20 infusion regimens, it became clear that the usual design of extension studies, which repeat pivotal study design over a longer study duration to collect long-term safety and efficacy data would not be a satisfactory method for investigating acceptability of higher infusion parameters than approved. To provide data qualifying for a label update, the study design instead required the application of a forced upward titration for every study participant. The range of volumes and flow rates in our study was selected based on extensive information from clinical studies and almost 8 years of market use of IgPro20, as well as other $20 \%$ SCIG products.

In addition to the investigation of infusion parameters higher than those currently approved, this is also the first clinical study of IgPro20 infusion using the manual push technique, which will evaluate several features of this technique in comparison with pump-assisted administration.

As a separate topic, there are currently very few infusion pumps for subcutaneous IgG products on the market that allow delivery of high volumes (more than $50-60 \mathrm{~mL}$ ) at high flow rates $(>100 \mathrm{~mL} / \mathrm{h}$ ). Future development of SCIG infusion techniques, especially with increasing use of IgG therapy for autoimmune conditions where IgG doses are significantly higher than in immune replacement, should be facilitated by new pump models that can deliver larger volumes at faster rates.

\section{Conclusion}

In conclusion, this study is the first prospective study that applied a new rigorous forced upward titration study design to evaluate individual safety and tolerability levels of pump-assisted and manual push SCIG infusion parameters. Results of this study may be used to seek regulatory approval for higher infusion SCIG parameters and new administration methods.

\section{Methods}

\section{Study design}

This was a multicenter, open-label, parallel-arm, non-randomized, study applying forced upward titration design to evaluate safety and tolerability of infusion parameters of IgPro20 at levels higher than those currently approved in patients with PID (NCT03033745). Patients were enrolled in one of the following 
three cohorts: Pump-assisted Volume Cohort, Pump-assisted Flow Rate Cohort and Manual Push Flow Rate Cohort. Each included patient meeting cohort-specific inclusion criteria, detailed below. The study contained a screening period of up to 28 days ( 4 weeks) followed by an active treatment period of 12 weeks in the Pump-assisted Volume Cohort and Manual Push Flow Rate Cohort, or 16 weeks in the Pump-assisted Flow Rate Cohort (Figure 1). The overall study duration (First Patient First Visit: 01 February 2017, Last Patient Last Visit: 14 December 2018) was 22.5 months.

Objectives and endpoints

The primary objective of the study was to determine responder rates under escalating infusion parameters. In the pump-assisted cohorts, a responder was defined as a patient who administered $\geq 3$ valid infusions at a given infusion parameter level. In the Manual Push Flow Rate Cohort, a responder was defined as a patient who administered the minimum prespecified number of valid infusions for a given infusion parameter level, as specified in Table 1.

\section{Table 1 Responder requirements in Manual Push Flow Rate Cohort}

\begin{tabular}{ccc}
\hline $\begin{array}{c}\text { Manual Push Infusions } \\
\text { per Week }\end{array}$ & Total Number of Infusions Scheduled for Parameter Level & $\begin{array}{c}\text { Minimum Number of } \\
\text { Valid Infusions }\end{array}$ \\
\hline 2 & 8 & 5 \\
3 & 12 & 7 \\
4 & 16 & 10 \\
5 & 20 & 12 \\
6 & 24 & 15 \\
7 & 28 & 17 \\
\hline
\end{tabular}

The escalating infusion parameters were as follows: Pump-assisted Volume Cohort: volume per injection site of 25, 40, and $50 \mathrm{~mL}$, Pump-assisted Flow Rate Cohort: flow rate per injection site of 25, 50, 75 and $100 \mathrm{~mL} / \mathrm{h}$ and Manual Push Flow Rate Cohort: flow rate per injection site of 30, 60, and $120 \mathrm{~mL} / \mathrm{h}(0.5,1$, and $2 \mathrm{~mL} / \mathrm{min}$, correspondingly; Figure 1). In pump-assited infusions, these constitute a volume two-fold higher and a flow rate four-fold higher than currently approved for IgPro20 in PID in the United States. The manual push technique is not currently approved. Secondary objectives were to evaluate the safety and 
tolerability of pump-assisted and manual push IgPro20 infusions at higher infusion parameters (volume and flow rate) than those currently approved. The assessment of serum IgG concentrations under various IgPro20 infusion parameters was an exploratory objective of the study.

An infusion was considered valid if $\geq 95 \%$ of the prescribed dose was used without any interruptions or decrease of the infusion parameter under test (including possible interruption for technical reasons) and where no prohibited concomitant medication was administered (Table 2).

\section{Table 2 Minimum criteria for a valid infusion}

\begin{tabular}{|c|c|}
\hline $\begin{array}{l}\text { Infusion } \\
\text { Parameter }\end{array}$ & $\begin{array}{l}\text { Definition Per Protocol } \\
\text { (Main Analysis) }\end{array}$ \\
\hline \multicolumn{2}{|c|}{ Pump-assisted Volume Cohort } \\
\hline Volume & $\begin{array}{l}\text { Maximum volume administered per injection site } \geq 95 \% \text { of the planned } \\
\text { maximum volume (ie, } 25,40 \text {, or } 50 \mathrm{~mL} \text { ) for } \geq 1 \text { injection site }\end{array}$ \\
\hline Dose & Total dose administered is $\geq 95 \%$ of the planned dose \\
\hline $\begin{array}{l}\text { Interruptions } \\
\text { or stops }\end{array}$ & $\begin{array}{l}\text { Not interrupted or stopped prematurely for any reason as documented in } \\
\text { the eDiary }\end{array}$ \\
\hline
\end{tabular}
Pump-assisted Flow Rate Cohort
Flow rate Injection site flow rate is $\geq 95 \%$ of the planned flow rate (ie, $25,50,75$, or $100 \mathrm{~mL} / \mathrm{h}$ ) for $\geq 1$ injection site

Dose $\quad$ Total dose administered is $\geq 95 \%$ of the planned dose

Interruptions Not interrupted or stopped prematurely for any reason as documented in or stops the eDiary

\begin{tabular}{ll}
\hline Manual Push Flow Rate Cohort \\
Volume & The actual infusion volume is $\geq 95 \%$ of the planned infusion volume for \\
that infusion day & \\
Flow rate & $\begin{array}{l}\text { The actual infusion duration is not more than } 1 \text { minute or } 10 \% \\
\text { (whichever is larger) longer than the calculated infusion duration based }\end{array}$ \\
& $\begin{array}{l}\text { on the desired flow rate (ie, } 0.5,1, \text { or } 2 \mathrm{~mL} / \mathrm{min} \text { ) and the actual volume } \\
\text { infused }\end{array}$
\end{tabular}

eDiary, electronic diary

The rate of total treatment-emergent adverse events (TEAEs) and local reactions per patient and per infusion as well as the severity, duration, and time of onset of local reactions, including tolerability of 
infusion parameters, ie, percentage of infusions without severe local reactions, were the secondary endpoints.

\section{IgPro20 infusions}

The first IgPro20 infusion was administered at the study site on Day 1 and every 4 weeks for first infusion of the next scheduled step (infusion parameter) and to capture any safety/concomitant medication information. All other infusions were administered at home until the last IgPro20 infusion at Week 12 (Week 16 in Pump-assisted Flow Rate Cohort).

The Pump-assisted Volume Cohort and the Manual Push Flow Rate Cohort each tested 3 infusion parameter levels for 4 weeks each over a total duration of 12 weeks. The Pumpassisted Flow Rate Cohort tested 4 infusion parameter levels for 4 weeks each for a total of 16 weeks. After 4 weeks on an infusion parameter level, responders were switched to the next infusion parameter level. Non-responders were allowed to continue for the remaining study duration at the highest previous infusion parameter level to collect further safety data. The IgPro20 infusion parameters in each cohort are shown in Figure 2.

\section{Patient population}

A minimum of 45 evaluable patients ( 15 per cohort) including $\geq 14$ (31\%) pediatric patients aged $\leq 17$ years and $\geq 9(20 \%)$ obese patients with body mass index $(B M I) \geq 30 \mathrm{~kg} / \mathrm{m}^{2}$ were planned to be enrolled in the study. Male and female patients with PID (e.g., with diagnosis of common variable immunodeficiency [CVID] or X-linked agammaglobulinemia [XLA]) were included in the study, if they abide by the following accepted infusion parameters with IgPro20 for $\geq 1$ month before Day 1 (first IgPro20 infusion in the study) in the respective cohorts:

- Pump-assisted Volume Cohort: IgPro20 at volumes of $25 \mathrm{~mL}$ per injection site and weekly volume of $\geq 50 \mathrm{~mL}$

- Pump-assisted Flow Rate Cohort: IgPro20 at a flow rate of $25 \mathrm{~mL} / \mathrm{h}$ per injection site

- Manual push Flow Rate Cohort: IgPro20 infusions at a flow rate of approximately $0.5 \mathrm{~mL} / \mathrm{min}$ (equivalent of 25 to $30 \mathrm{~mL} / \mathrm{h}$ ) per injection site. 
Patients with hypersensitivity to IgPro20, ongoing serious bacterial infections at the time of screening or with other significant medical conditions, and who received therapy or were under drug or medication abuse $\leq 1$ year before the study were excluded from the study. The patients were assigned to a cohort based on investigators discretion and not randomized.

\section{Efficacy assessments}

No clinical efficacy variables other than serum IgG levels were assessed in this study due to its short duration per patient. Serum IgG trough concentrations were determined at Day 1 and at End of Study Visit at the study site as a surrogate parameter for efficacy.

\section{Safety assessments}

\section{AEs, tolerability, and other analyses}

AEs starting on or after the date (and time if available) of the first in-study administration of IgPro20 were considered as TEAEs. AEs which started during infusion or within 72 hours after the end of an infusion were considered temporally associated AEs. For non-responder patients at a given infusion parameter level, AEs starting at or after the next infusion following the infusion leading to non-response, were analyzed separately from the AEs reported before that time to adequately characterize safety under the forced upward titration conditions.

Tolerability was defined as the number of infusions without severe local reactions divided by the total number of infusions, irrespective of their validity i.e., a tolerability of 1.00 corresponds to the most favorable tolerability (no severe local reactions) and a tolerability of 0 corresponds to the most unfavorable tolerability (severe local reactions for every infusion). All TEAEs, tolerability data and the extent of exposure were summarized by cohort and infusion parameter level.

Clinical laboratory evaluations (hematology, biochemistry) and vital signs were also analyzed.

\section{Statistical methods}

All analyses were performed in the safety analysis set, which comprised all patients who received at least a partial dose of IgPro20 in the study. Responder rates, number of total infusions, and number and percentage of valid infusions were calculated for each infusion parameter level. Results were summarized overall, and by age ( $\leq 17$ years, $>17$ years) and BMI $\left(<30 \mathrm{~kg} / \mathrm{m}^{2}, \geq 30 \mathrm{~kg} / \mathrm{m}^{2}\right)$. The responder rate for each cohort was calculated as the number of patients who successfully completed an infusion 
parameter as a proportion of all patients included in the safety analysis set. An infusion parameter level was deemed "successful" if the responder rate was $\geq 33 \%$.

Serum IgG trough concentrations were summarized descriptively by cohort. In addition to the responder analysis, two sensitivity analyses were conducted for the pump-assisted cohorts considering infusions that were stopped or interrupted for technical reasons as valid, as long as no related AEs started during the interrupted or stopped infusions. Local reactions (including time of onset and duration), other TEAEs, clinical laboratory evaluations (hematology, biochemistry) and vital signs were summarized descriptively by infusion parameter level.

\section{Abbreviations}

AEs, BMI, CVID, IgG, IVIG, PID, SCIG, TEAEs, XLA

\section{Declarations}

\section{Ethics approval and consent to participate}

The clinical study protocol and all amendments were approved by the independent ethics committees/institutional review boards of the participating centers. This study was carried out in accordance with the International Council for Harmonisation (ICH) Good Clinical Practice (GCP) guidelines and in accordance with Declaration of Helsinki. Patient informed consent was obtained and documented according to the provisions of ICH GCP and applicable regulatory requirements. For patients aged $<16$ years, written informed consent to participate in the study was obtained from a parent or legal guardian.

\section{Consent for publication}

Not applicable

\section{Competing Interests}

MAR, JH and MP are employees of CSL Behring and own CSL Behring shares.

\section{Funding}

This study is funded by CSL Behring, who designed the study, collected, analyzed, and interpreted the data and funded editorial and graphical support from Fishawack Communications $\mathrm{GmbH}$, Basel, Switzerland, a member of the Fishawack group of companies. 


\section{Availability of Data and Materials}

The study protocol and results are available on clinicaltrials.gov

(https://clinicaltrials.gov/ct2/show/NCT03033745)

\section{Authors' Contributions}

MAR, JH, and MP have read, reviewed, and approved the manuscript and have contributed substantially to the work described.

\section{Acknowledgments}

Editorial and graphical support was provided by Vibhuti Singh, PhD and Heather Shawcross, PhD of Fishawack Communications $\mathrm{GmbH}$, Basel, Switzerland, a member of the Fishawack Group of Companies, supported by CSL Behring. The data summarized in this study are from a CSL Behring-sponsored clinical trial.

\section{References}

1.Berger M. Principles of and advances in immunoglobulin replacement therapy for primary immunodeficiency. Immunol Allergy Clin North Am. 2008;28(2):413-37.

2.Gardulf A, Nicolay U, Math D, Asensio O, Bernatowska E, Bock A, et al. Children and adults with primary antibody deficiencies gain quality of life by subcutaneous IgG self-infusions at home. J Allergy Clin Immunol. 2004;114(4):936-42.

3.Jolles S, Orange JS, Gardulf A, Stein MR, Shapiro R, Borte M, et al. Current treatment options with immunoglobulin $\mathrm{G}$ for the individualization of care in patients with primary immunodeficiency disease. Clin Exp Immunol. 2015;179(2):146-60.

4.Wasserman RL, Melamed I, Nelson RP, Jr., Knutsen AP, Fasano MB, Stein MR, et al. Pharmacokinetics of subcutaneous IgPro20 in patients with primary immunodeficiency. Clin Pharmacokinet. 2011;50(6):40514.

5.Shapiro R. Subcutaneous immunoglobulin therapy by rapid push is preferred to infusion by pump: a retrospective analysis. J Clin Immunol. 2010;30(2):301-7.

6.Bienvenu B, Cozon G, Mataix Y, Lachaud D, Alix A, Hoarau C, et al. Rapid Push vs Pump-Infused Subcutaneous Immunoglobulin Treatment: a Randomized Crossover Study of Quality of Life in Primary Immunodeficiency Patients. J Clin Immunol. 2018;38(4):503-12. 
7.Pac M, Bernatowska E. Rapid push: new opportunities in subcutaneous immunoglobulin replacement therapy. Central European Journal of Immunology. 2013;38 (3)(388-392.).

8.Sidhu J, Rojavin M, Pfister M, Edelman J. Enhancing Patient Flexibility of Subcutaneous Immunoglobulin G Dosing: Pharmacokinetic Outcomes of Various Maintenance and Loading Regimens in the Treatment of Primary Immunodeficiency. Biol Ther. 2014;4(1-2):41-55.

9.Shapiro RS. Subcutaneous immunoglobulin: rapid push vs. infusion pump in pediatrics. Pediatr Allergy Immunol. 2013;24(1):49-53.

10.Shapiro RS. Subcutaneous immunoglobulin therapy given by subcutaneous rapid push vs infusion pump: a retrospective analysis. Ann Allergy Asthma Immunol. 2013;111(1):51-5.

11.Shapiro R. Subcutaneous immunoglobulin (16 or $20 \%$ ) therapy in obese patients with primary immunodeficiency: a retrospective analysis of administration by infusion pump or subcutaneous rapid push. Clin Exp Immunol. 2013;173(2):365-71.

12.Cozon GJN, Clerson P, Dokhan A, Fardini Y, Sala TP, Crave JC. In-depth interviews of patients with primary immunodeficiency who have experienced pump and rapid push subcutaneous infusions of immunoglobulins reveal new insights on their preference and expectations. Patient Prefer Adherence. 2018;12:423-9.

13.Jolles S, Sleasman JW. Subcutaneous immunoglobulin replacement therapy with Hizentra, the first 20\% SCIG preparation: a practical approach. Adv Ther. 2011;28(7):521-33.

14.Shehata N, Palda V, Bowen T, Haddad E, Issekutz TB, Mazer B, et al. The Use of Immunoglobulin Therapy for Patients With Primary Immune Deficiency: An Evidence-Based Practice Guideline. Transfus Med Rev. 2010;24(1):S28-S50.

15.Patel NC, Gallagher JL, Ochs HD, Prescott Atkinson T, Wahlstrom J, Dorsey M, et al. Subcutaneous Immunoglobulin Replacement Therapy with Hizentra(R) is Safe and Effective in Children Less Than 5 Years of Age. J Clin Immunol. 2015;35(6):558-65.

16.Espanol T, Prevot J, Drabwell J, Sondhi S, Olding L. Improving current immunoglobulin therapy for patients with primary immunodeficiency: quality of life and views on treatment. Patient Prefer Adherence. 2014;8:621-9.

17.Petersson C, Fust R, Hagstedt C, Vagstrom P, Nilsdotter-Augustinsson A. "Experiences of the burden of treatment" - patient-reports of facilitated subcutaneous immunoglobulin treatment in adults with immunodeficiency. Journal of clinical nursing. 2018.

18.Jolles $\mathrm{S}$. Hyaluronidase facilitated subcutaneous immunoglobulin in primary immunodeficiency. Immunotargets Ther. 2013;2:125-33. 
19.Martin A, Lavoie L, Goetghebeur M, Schellenberg R. Economic benefits of subcutaneous rapid push versus intravenous immunoglobulin infusion therapy in adult patients with primary immune deficiency. Transfus Med. 2013;23(1):55-60.

20.Misbah S, Sturzenegger MH, Borte M, Shapiro RS, Wasserman RL, Berger M, et al. Subcutaneous immunoglobulin: opportunities and outlook. Clin Exp Immunol. 2009;158 Suppl 1:51-9.

21.Paris K, Haddad E, Borte M, Brodszki N, Derfalvi B, Marodi L, et al. Tolerability of subcutaneous immunoglobulin $20 \%$, Ig20Gly, in pediatric patients with primary immunodeficiencies. Immunotherapy. 2019;11(5):397-406.

22.Ponsford M, Carne E, Kingdon C, Joyce C, Price C, Williams C, et al. Facilitated subcutaneous immunoglobulin (fSClg) therapy-practical considerations. Clin Exp Immunol. 2015;182(3):302-13.

23.Schmidt RE, Ochs HD. 7th International Immunoglobulin Conference: Immunodeficiencies. Clin Exp Immunol. 2014;178 Suppl 1:21.

24.Wasserman RL, Melamed I, Stein MR, Gupta S, Puck J, Engl W, et al. Recombinant human hyaluronidase-facilitated subcutaneous infusion of human immunoglobulins for primary immunodeficiency. J Allergy Clin Immunol. 2012;130(4):951-7 e11.

25.Jolles S, Borte M, Nelson RP, Jr., Rojavin M, Bexon M, Lawo JP, et al. Long-term efficacy, safety, and tolerability of Hizentra(R) for treatment of primary immunodeficiency disease. Clin Immunol. 2014;150(2):161-9.

26.Kanegane H, Imai K, Yamada M, Takada H, Ariga T, Bexon M, et al. Efficacy and safety of IgPro20, a subcutaneous immunoglobulin, in Japanese patients with primary immunodeficiency diseases. J Clin Immunol. 2014;34(2):204-11.

27.van Schaik IN, Bril V, van Geloven N, Hartung HP, Lewis RA, Sobue G, et al. Subcutaneous immunoglobulin for maintenance treatment in chronic inflammatory demyelinating polyneuropathy (PATH): a randomised, double-blind, placebo-controlled, phase 3 trial. Lancet Neurol. 2018;17(1):35-46.

28.European Medicines Agency (EMA). Guideline on the clinical investigation of human normal immunoglobulin for subcutaneous and/or intramuscular administration (SClg/IMlg). 2015 [Available from: https://www.ema.europa.eu/en/documents/scientific-guideline/guideline-clinical-investigationhuman-normal-immunoglobulin-subcutaneous/intramuscular-administration-scig/imig_en.pdf.

29.Arias Pou P, Gonzalez A, Martinez LJP, Latorre AD, Alonso MS. Periprocedural management of patients receiving novel oral anticoagulants. European journal of hospital pharmacy Science and practice. 2018;25(6):292-7.

30.Food and Drug Administration (FDA). Guidance for Industry: Safety, Efficacy, and Pharmacokinetic Studies to Support Marketing of Immune Globulin Intravenous (Human) as Replacement Therapy for 
Primary Humoral Immunodeficiency 2008 [Available from:

https://www.fda.gov/media/124333/download.

31.Pasquet M, Pellier I, Aladjidi N, Auvrignon A, Cherin P, Clerson P, et al. A cohort of French pediatric patients with primary immunodeficiencies: are patient preferences regarding replacement immunotherapy fulfilled in real-life conditions? Patient Prefer Adherence. 2017;11:1171-80.

\section{Figures}

PubMed and EMBASE database searches

"subcutaneous immunoglobulin" or

"subcutaneous immune globulin" or

"SCIG" or "IGSC" or "manual administration"

or "rapid push" or "manual" and

"primary immunodeficiency" or

"primary immune deficiency"

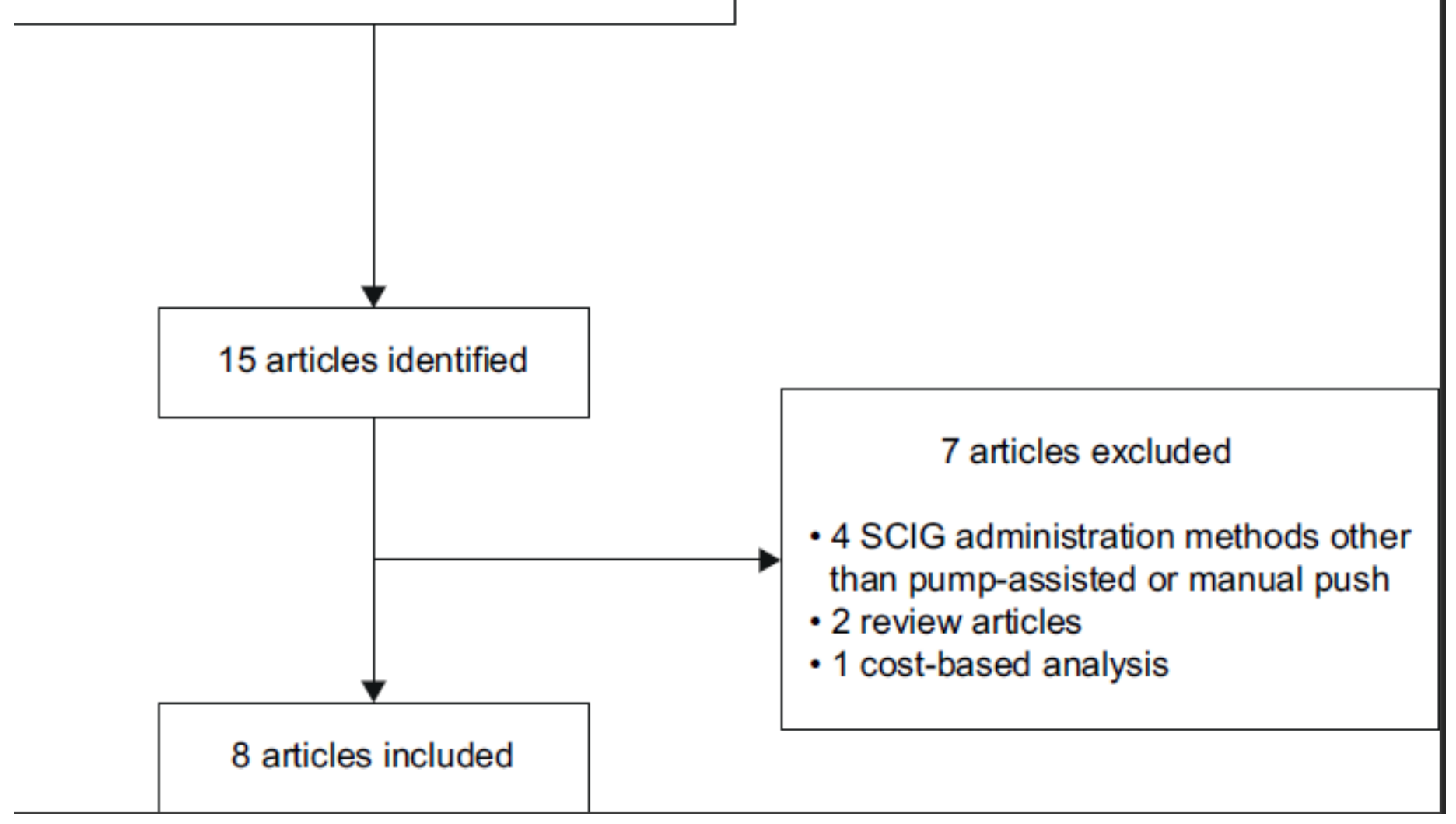

\section{Figure 1}

Flowchart for systemic literature search IGSC, subcutaneous immunoglobulin G; SCIG, subcutaneous immunoglobulin G; PID, primary immunodeficiency 


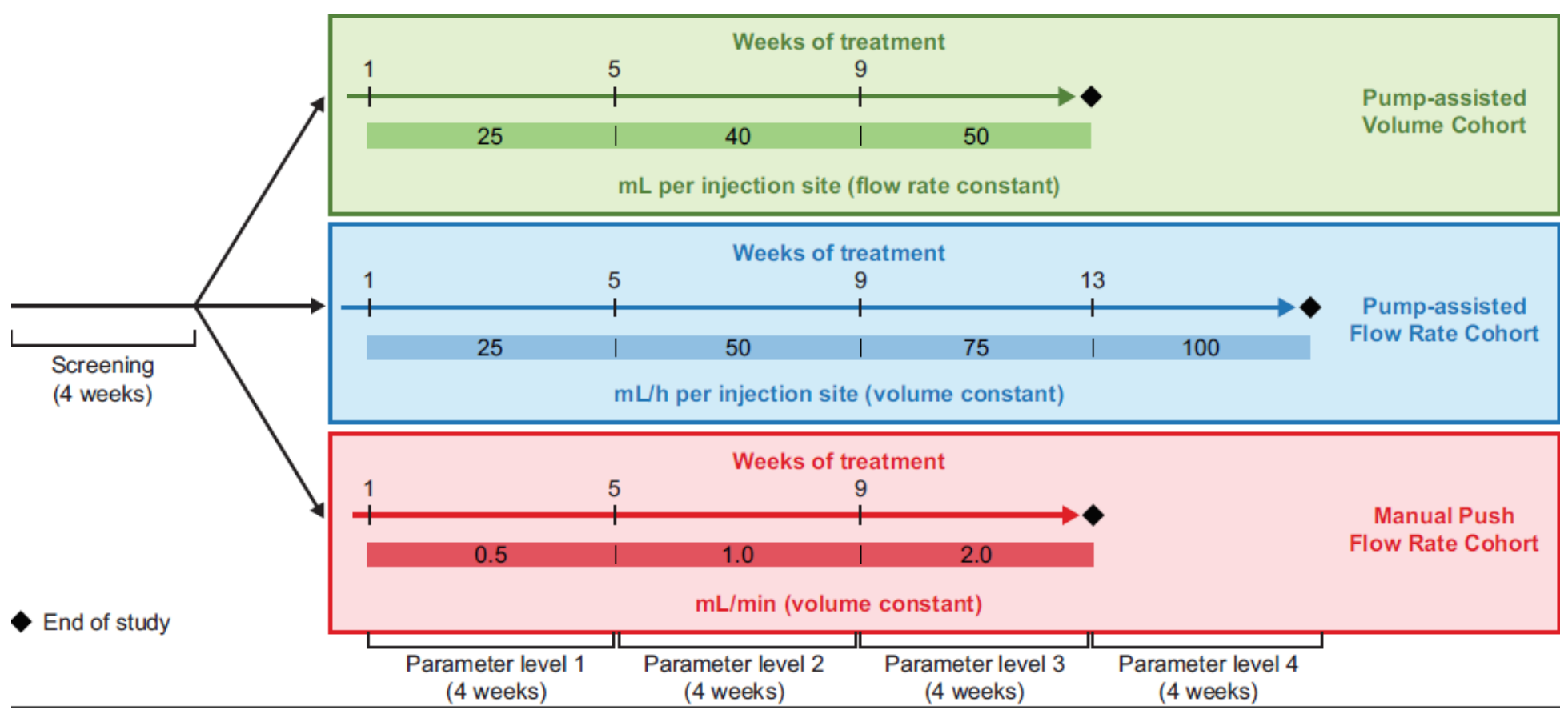

Figure 2

Study design

\section{Supplementary Files}

This is a list of supplementary files associated with this preprint. Click to download.

- Individualpatientdatasharingstatement.docx 